\title{
Expression of AMPK, SIRT1, and ACC Differs between Winter- and Summer-Acclimatized Djungarian Hamsters
}

\author{
Sanni M. E. Kinnunen ${ }^{1, \star}$ \\ Satu K. Mänttäri ${ }^{2}$ \\ Seppo Y. O. Saarela ${ }^{1}$ \\ ${ }^{1}$ Department of Genetics and Physiology, University of Oulu, \\ PO Box 3000, FI-90014 Oulu, Finland; ${ }^{2}$ Finnish Institute of \\ Occupational Health, Aapistie 1, FI-90220 Oulu, Finland
}

Accepted 8/6/2017; Electronically Published 9/7/2017

\begin{abstract}
The wintering strategy of the Djungarian hamster (Phodopus sungorus) includes a naturally occurring decrease in food intake and body mass. Our aim was to investigate the conceivable role of the metabolic regulators, AMP-activated protein kinase (AMPK) and sirtuin 1 (SIRT1), in the seasonal adaptation of the Djungarian hamster. In addition, a rate-limiting enzyme in fatty acid synthesis and oxidation, acetyl CoA carboxylase (ACC), was studied. Relative protein expressions and phosphorylated forms (pAMPK and pACC) were determined by Western blot from subcutaneous white adipose tissues (sWAT), abdominal white adipose tissues (aWAT), interscapular brown adipose tissues (iBAT), skeletal muscle, and hypothalamus of winter- and summer-acclimatized hamsters. The winter group had higher AMPK expression in sWAT, aWAT, and iBAT, but the relative amount of phosphorylated protein (pAMPK/AMPK ratio) was lower in these tissues. Furthermore, ACC expression was higher in sWAT and iBAT of the winter animals. pACC (inactive form) levels were higher in all adipose tissues, yet a lower pACC/ACC ratio was detected in $\mathrm{BBAT}$ of the winter hamsters. Muscle AMPK expression was lower but pAMPK/AMPK ratio higher in the winter group. SIRT1 expression was higher in muscle and all adipose tissues of the winter hamsters. Hypothalamic protein expressions did not differ between the groups. Higher expressions of AMPK, ACC, and SIRT1 in WAT and iBAT of the winter hamsters suggest a role in the regulation of lipid reserves and increased thermogenic capacity characteristic to the winter-adapted Djungarian hamsters.
\end{abstract}

Keywords: body mass, energy metabolism, seasonal adaptation, white adipose tissue, brown adipose tissue, Siberian hamster.

*Corresponding author; e-mail: sanni.kinnunen@oulu.fi.

Physiological and Biochemical Zoology 90(6):605-612. 2017. (C) 2017 by The University of Chicago. All rights reserved. 1522-2152/2017/9006-17023\$15.00. DOI: $10.1086 / 694295$

\section{Introduction}

Seasonal changes in food intake, body weight, and adiposity are common for mammals living at northern latitudes where food resources are limited during winter. The autumnal accumulation of body fat stores and hibernation throughout the winter is a survival strategy especially for many small species. However, some species have quite the opposite physiological response before winter. The Djungarian hamster (Phodopus sungorus), also known as the Siberian hamster, is a photoperiodic species that voluntarily reduces energy intake approximately $20 \%-30 \%$ when exposed to a short photoperiod despite ad lib. access to food (Heldmaier and Steinlechner 1981a; Wade and Bartness 1984; Knopper and Boily 2000). Wintertime body mass of the animals can be up to $30 \%-40 \%$ lower compared to the long photoperiod, with most of the lost mass being adipose tissue (Heldmaier and Steinlechner 1981a; Klingenspor et al. 2000). Thus, the energy required for the maintenance of body mass is reduced and consequently the needed amount of food and foraging activity is decreased. In addition, Djungarian hamsters display daily torpor as an energy-saving strategy (Heldmaier and Steinlechner 1981a, 1981b). This naturally occurring reduction in body adiposity offers an interesting model to study molecular mechanisms that participate in seasonal metabolic adjustments.

One key enzyme in the control of energy metabolism is AMPactivated protein kinase (AMPK). It is a cellular energy and nutrient sensor that is activated in response to decreased energy levels in the cell (Hardie et al. 2012). Moreover, AMPK has a broad role in the maintenance of whole-body energy balance, including the hypothalamic control of food intake and appetite as well as the regulation of glucose and lipid metabolism in the peripheral tissues (Andersson et al. 2004; Minokoshi et al. 2004; Mulligan et al. 2007). AMPK regulates the expression and activity of numerous metabolic enzymes. For instance, AMPK modulates lipid metabolism through acetyl CoA carboxylase (ACC), a rate-limiting enzyme in fatty acid (FA) synthesis and oxidation. AMPK inhibits ACC by phosphorylation, which consequently increases mitochondrial $\beta$-oxidation and decreases FA synthesis (Kahn et al. 2005). Another essential metabolic regulator is sirtuin 1 (SIRT1), a member in the family of mammalian $\mathrm{NAD}^{+}$-dependent histone/protein deacetylases (SIRT17). SIRT1 expression and activity have been shown to increase under conditions of reduced nutrient availability, such as fasting and calorie restriction. SIRT1 has numerous target substrates, including regulators of metabolic pathways related to lipid metabolism and mitochondrial biogenesis (Rodgers et al. 2005; Gerhart-Hines et al. 2007). In general, AMPK and SIRT1 are activated in response to changes in energy balance. As a result, the 
energy-producing pathways are enhanced and energy-consuming processes are downregulated (Fulco and Sartorelli 2008). In addition to common activators, such as fasting, calorie restriction, and exercise, AMPK and SIRT1 have overlapping functions in cellular energy metabolism. They have common target molecules in glucose and lipid metabolism, such as peroxisome proliferatoractivated receptor gamma coactivator- $1 \alpha(\mathrm{PGC}-1 \alpha)$, a transcriptional coregulator that promotes mitochondrial biogenesis (Haigis and Sinclair 2010; Ruderman et al. 2010).

Previous studies in hibernating species suggest involvement of AMPK and SIRT1 in the metabolic adjustments during seasonal adaptation. In marmots (Marmota flaviventris) and ground squirrels (Callospermophilus lateralis) hypothalamic AMPK participates in the regulation of food intake during hibernation season (Florant et al. 2010; Healy et al. 2011a). In peripheral tissues, such as white adipose tissues (WAT) and brown adipose tissues (BAT) and liver, AMPK expression and phosphorylation vary in response to different stages of the torpor bout and also between the summer- and winteracclimatized euthermic ground squirrels (C. lateralis and Ictidomys tridecemlineatus; Horman et al. 2005; Healy et al. 2011b; Lanaspa et al. 2015). Furthermore, tissue-specific alterations of expression levels and activity of different sirtuins were observed during the torpor-arousal cycle of the ground squirrel (I. tridecemlineatus; Rouble and Storey 2015). However, data supporting a role for AMPK and SIRT1 in seasonally occurring metabolic changes mainly come from studies in species exhibiting autumnal fattening, total aphagia, and deep hibernation in winter. The diverse wintering strategy of the Djungarian hamster presents a comparative model that could reveal further information about the function of these proteins in the seasonal regulation of energy metabolism. In this study, we evaluated AMPK expression and phosphorylation (active form) level and SIRT1 expression in different tissues of winterand summer-acclimatized hamsters. In addition, the expression and phosphorylation of ACC, a downstream target of AMPK, were analyzed. We hypothesized that due to their central roles in the metabolic processes, especially in lipid metabolism, these proteins are involved in the maintenance of energy homeostasis in the lean winter phenotype of the Djungarian hamster and are differentially expressed in winter- and summer-acclimatized animals.

\section{Material and Methods}

Djungarian hamsters (age 12-13 mo; 16 males and 8 females) were obtained from the breeding colony at the Department of Biology, University of Oulu. Before the experiment, the hamsters were housed individually in an animal room under a $12 \mathrm{~L}: 12 \mathrm{D}$ cycle and at an ambient temperature of $22^{\circ} \pm 2^{\circ} \mathrm{C}$. Animals had free access to water and food (Lactamin) and weekly supplement of apple and curd. Animals used in this study received the same diet throughout the experiment.

The experimental protocol was approved by Finland's national Animal Experiment Board (license no. ESAVI-201006711/Ym-23). Hamsters were randomly divided into two groups: winter acclimatization $(N=16 ; 11$ males and 5 females) and summer acclimatization $(N=8 ; 5$ males and $3 \mathrm{fe}$ males). Winter animals were exposed to a short photoperiod (8L: $16 \mathrm{D}$, lights on at 0800 hours) at $10^{\circ} \mathrm{C}$, and summer animals were housed in a long photoperiod (14L:10D, lights on at 0600 hours) at $22^{\circ} \mathrm{C}$ for $15 \mathrm{wk}$ (December 30 -April 13). Hamsters were weighed weekly throughout the experimental period, and incidence of possible torpor bouts was monitored visually every other day. At the end of the experiment, hamsters were killed during the light phase (between 0900 and 1500 hours) by cervical dislocation in a nonfasted and nontorpid state. Whole hypothalamus, hind limb muscle ( $m$. rectus femoris), samples from subcutaneous (inguinal) and abdominal (retroperitoneal) white adipose tissues (sWAT and aWAT), and interscapular brown adipose tissue (iBAT) were collected and frozen immediately in liquid nitrogen and stored at $-80^{\circ} \mathrm{C}$ for later analyses.

Tissue samples and whole hypothalamus were prepared, and Western blot analysis was conducted as previously described (Kinnunen et al. 2016). Briefly, samples ranging from 20 to $150 \mathrm{mg}$ were homogenized in 6 vol ice-cold buffer, and the insoluble material was removed by centrifugation $(17,000 \mathrm{~g}$ for $10 \mathrm{~min}$ ). Samples containing an equal amount of protein were resolved by SDS-PAGE and transferred to nitrocellulose membrane. The total amount of protein loaded into each well of the gels was $10 \mu \mathrm{g}$ for hypothalamus, $12 \mu \mathrm{g}$ for muscle, $16 \mu \mathrm{g}$ for iBAT, and $20 \mu \mathrm{g}$ for sWAT and aWAT. Membranes were incubated overnight at $+4^{\circ} \mathrm{C}$ in primary antibodies against pAMPK $\alpha$ (Thr172) 2535, AMPK $\alpha$ 2532, pACC (Ser79) 3661, ACC 3662 (Cell Signaling Technology, Beverly, MA), and SIRT1 12193 (Abcam, Cambridge, UK). The antibodies for pACC and ACC recognize both isoforms of ACC: ACC1 $(265 \mathrm{kDa})$ and ACC2 $(280 \mathrm{kDa})$. Anti- $\beta$-actin ab8227 (Abcam) or anti- $\alpha$ tubulin 2125 (Cell Signaling Technology) antibodies were used as loading controls. Antibodies were validated for the Djungarian hamster using rat and mouse samples as positive controls. All antibodies gave corresponding bands at the expected molecular masses in the hamster samples. AP-conjugated goat anti-rabbit (Bio-Rad Laboratories) was used as secondary antibody. Antibody detection was performed with NBT/BCIP stock solution (Roche Diagnostics). Optical densities of the detected bands were analyzed with the FluorS MultiImager program (Bio-Rad). Results were normalized with the corresponding loading control.

All statistical analyses were performed using SPSS v20.0 for Windows (IBM). The Shapiro-Wilk test was used to test normality, and homogeneity of variances was tested by the Levene test. Comparisons between the groups were performed using the Student's $t$-test. Correlations were calculated with the Pearson correlation coefficient $\left(r_{\mathrm{p}}\right)$. Statistical significance was defined as $P<0.05$. The results are presented as means \pm SEM.

\section{Results}

Hamsters lost weight progressively in response to winter acclimatization, and body weight remained stable for the last $2 \mathrm{wk}$ of the experiment, indicating that the animals had reached 


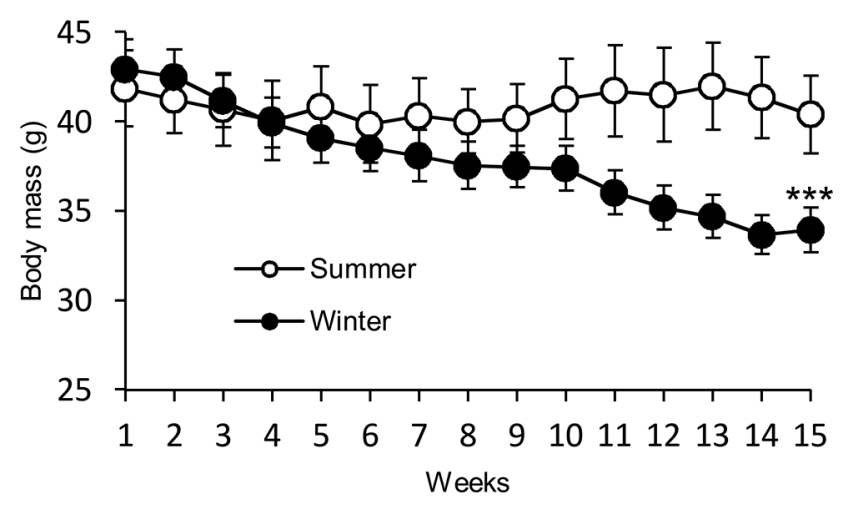

Figure 1. Average body masses of the hamsters during the 15 -wk experimental period. Winter-acclimatized Djungarian hamsters $(N=$ 16; filled circles) lost $21.4 \% \pm 2.1 \%(9.7 \pm 1.2 \mathrm{~g})$ of their body mass. Asterisks indicate significant difference between body mass at the start and end of the experiment (paired-sample Student's $t$-test: three asterisks, $P<0.001)$. The body mass of the summer group $(N=8$; open circles) did not change significantly.

their body fat nadir (fig. 1). After the 15-wk experimental period, the winter group had lost $21.4 \% \pm 2.1 \%(9.7 \pm 1.2 \mathrm{~g})$ of their body mass $(P<0.001)$, but the body mass of the summer group did not change significantly. Occurrence of regular daily torpor was evident in only two individuals. Torpid animals were curled in their dens and did not respond to external stimulation.

In sWAT, expression of AMPK was twofold higher in the winter group compared to the summer animals $(P<0.05)$, but the pAMPK level declined in the winter group $(P<0.05)$ and, consequently, the phosphorylation ratio was threefold lower than in the summer group $(P<0.05$; fig. $2 A)$. Both ACC and pACC levels were higher (1.5-fold, $P<0.05$, and twofold, $P<0.001$, respectively) in the winter animals, and also the pACC/ACC ratio was significantly higher in the winter group $(P<0.05$; fig. $2 B)$. There was no correlation between pAMPK and pACC. The SIRT1 level was 1.5 -fold higher in the winter animals $(P<0.05$; fig. 2C).

Also in aWAT, total AMPK expression was twofold higher in the winter animals $(P<0.05$; fig. $3 A)$. Although there were no statistically significant differences in the pAMPK level, the pAMPK/AMPK ratio was lower in the winter group, similar to sWAT (fig. 3A). ACC expression did not differ between the groups. The pACC level was 2.3 -fold higher in the winter animals, yet there was no statistical difference in the pACC/ACC ratio $(P=0.07$; fig. $3 B)$. Positive correlation was observed between $\mathrm{pAMPK}$ and pACC $\left(r_{\mathrm{p}}=0.519 ; P<0.05\right)$. SIRT1 protein expression was 2.4-fold higher in the winter group $(P<0.05$; fig. 3C).

In iBAT, AMPK expression was threefold higher in the winter group than in the summer group $(P<0.05$; fig. $4 A)$. No changes were observed in the pAMPK levels, and the pAMPK/ AMPK ratio was twofold lower in the winter animals $(P<0.05$; fig. $4 A$ ). Also the ACC expression was higher (fivefold) in the winter group $(P<0.001)$, with concomitant twofold elevation in the pACC levels $(P<0.05)$, but the pACC/ACC ratio was two- fold lower in the winter animals compared to the summer group $(P<0.05$; fig. $4 B)$. There was no correlation between pAMPK and pACC. SIRT1 expression was 1.7-fold higher in the winter group $(P<0.05$; fig. $4 C)$.

In the skeletal muscle, AMPK concentration was lower in the winter group than in the summer animals $(P<0.01$; fig. $5 A)$. The difference in the $\mathrm{pAMPK}$ level was not statistically significant, but the pAMPK/AMPK ratio was twofold higher $(P<0.05)$ in the winter group (fig. $5 A$ ). No significant differences were detected in the total ACC, pACC, or pACC/ACC ratio between the groups (fig. $5 B$ ). No correlation between AMPK and ACC phosphorylation was observed. SIRT1 expression was 1.5 -fold

\section{sWAT relative protein concentrations}
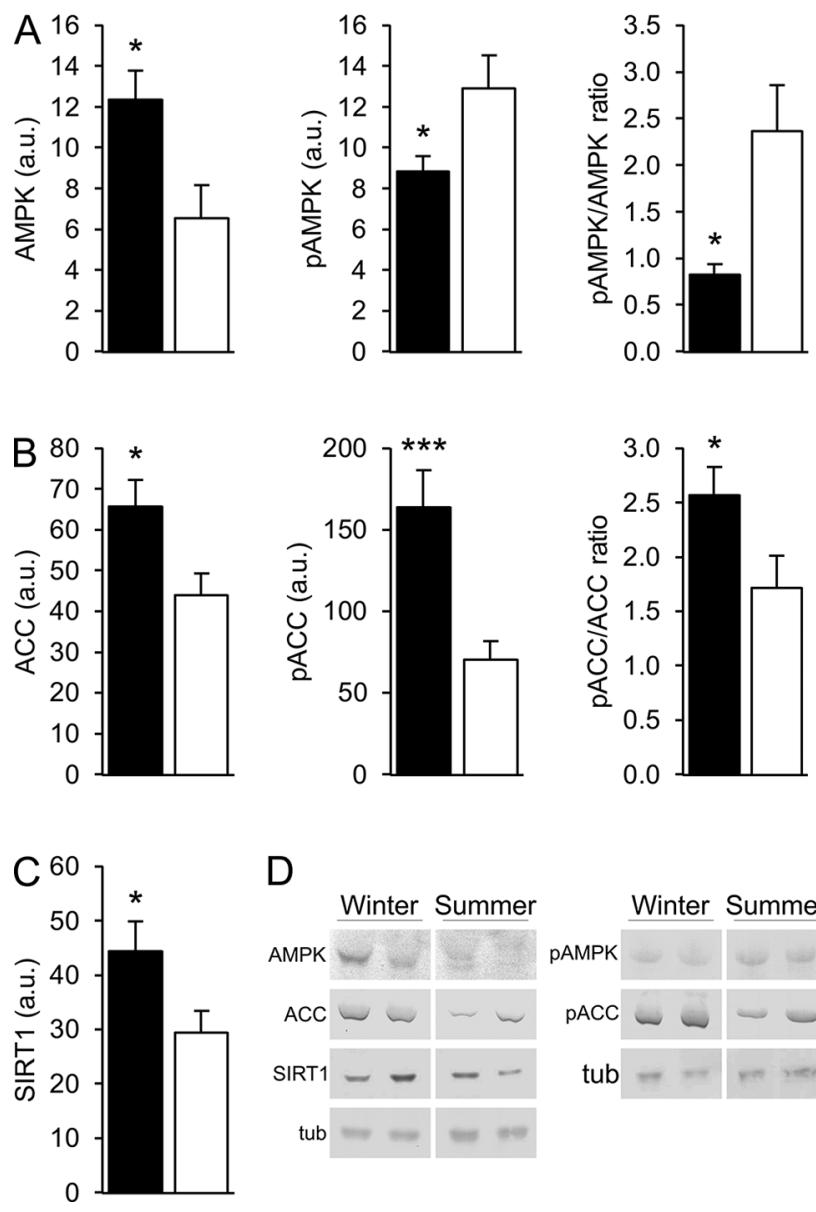

D

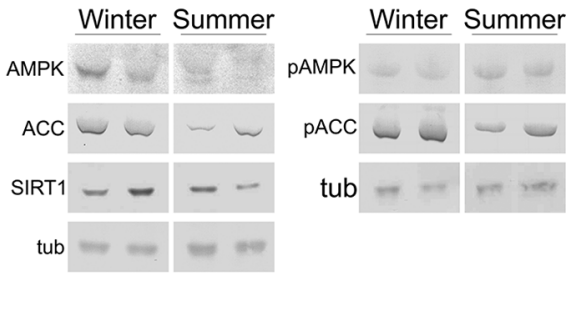

Figure 2. Expression of AMPK, ACC, and SIRT1 in the sWAT of winter- and summer-acclimatized Djungarian hamsters. Relative protein concentrations of total AMPK, phosphorylated AMPK (pAMPK), and pAMPK/AMPK ratio $(A)$; total ACC, phosphorylated ACC, and pACC/ACC ratio (B); and total SIRT1 $(C)$. D, Representative Western blots for AMPK, pAMPK, ACC, pACC, SIRT1, and loading control $\alpha$ tubulin (tub). The results are expressed as mean arbitrary units (a.u.) + SEM of protein band intensities normalized to $\alpha$-tubulin. Asterisks indicate significant differences between the groups (two-tailed Student's $t$-test: one asterisk, $P<0.05$; three asterisks, $P<0.001) . N=16$ for the winter group (filled bars) and $N=8$ for the summer group (open bars). 


\section{aWAT relative protein concentrations}
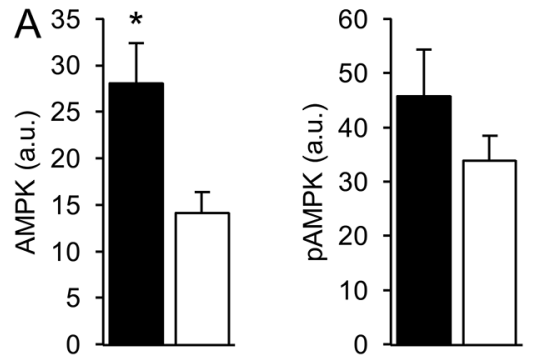

- Winter asummer
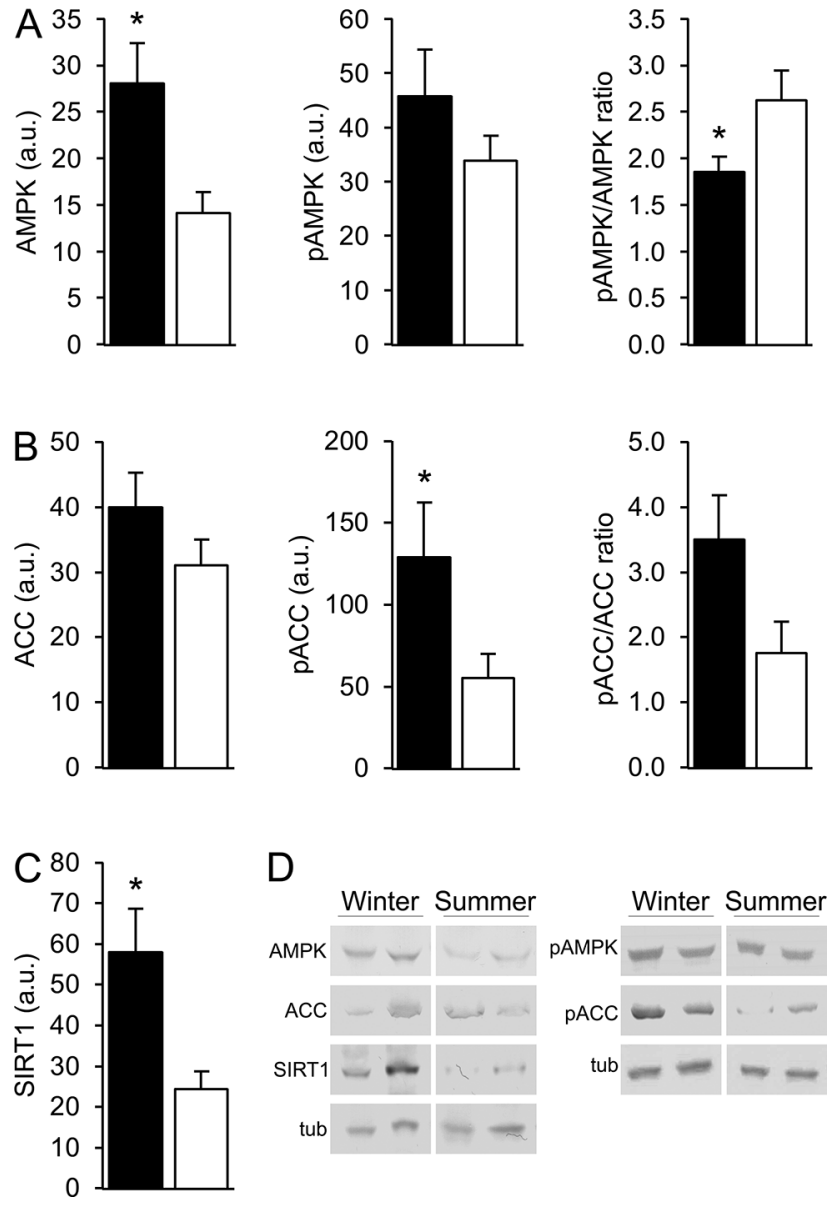

Figure 3. Expression of AMPK, ACC, and SIRT1 in the aWAT of winter- and summer-acclimatized Djungarian hamsters. Relative protein concentrations of total AMPK, phosphorylated AMPK (pAMPK), and pAMPK/AMPK ratio $(A)$; total ACC, phosphorylated ACC, and pACC/ACC ratio $(B)$; and total SIRT1 $(C)$. $D$, Representative Western blots for AMPK, pAMPK, ACC, pACC, SIRT1, and loading control $\alpha$ tubulin (tub). The results are expressed as mean arbitrary units (a.u.) + SEM of protein band intensities normalized to $\alpha$-tubulin. Asterisks indicate significant differences between the groups (two-tailed Student's $t$-test: one asterisk, $P<0.05$ ). $N=16$ for the winter group (filled bars) and $N=8$ for the summer group (open bars).

higher in the muscle of the winter group $(P<0.05$; fig. $5 C)$. There were no differences in hypothalamic AMPK, ACC, or SIRT1 expression or phosphorylation between the groups (fig. 6A-6C).

\section{Discussion}

In this study, we examined the relative protein expression levels of the metabolic regulators AMPK, ACC, and SIRT1 in sWAT, aWAT, iBAT, hypothalamus, and skeletal muscle of winterand summer-acclimatized Djungarian hamsters. The effect of winter acclimatization was observed in sWAT, aWAT, and BAT, where AMPK, SIRT1, and ACC protein expression was up to fivefold higher. These results indicate that AMPK, ACC, and
SIRT1 have a role in the seasonal metabolic adaptation of the Djungarian hamster.

Short day length has been shown to induce adipocyte lipolysis in the WAT depots of the Djungarian hamster (Demas and Bartness 2001; Demas et al. 2002; Bowers et al. 2005). We observed higher SIRT1 and AMPK expression in sWAT and aWAT of the winter hamsters compared to the summer animals. Conversely, AMPK phosphorylation ratios, that is, proportions of active protein, were lower in both tissues of the winter group. However, although phosphorylation is a good indicator of AMPK activity (Suter et al. 2006), it has to be noted that we did not measure enzyme activity per se. Both AMPK and

\section{BAT relative protein concentrations}
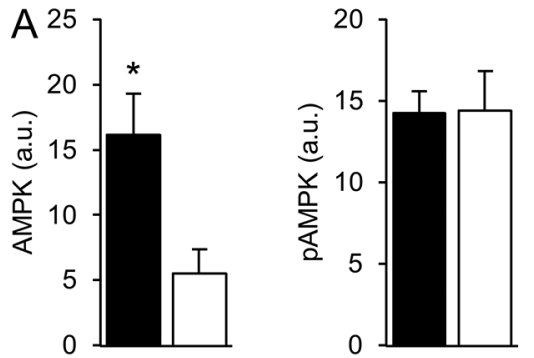

-Winter 口Summer
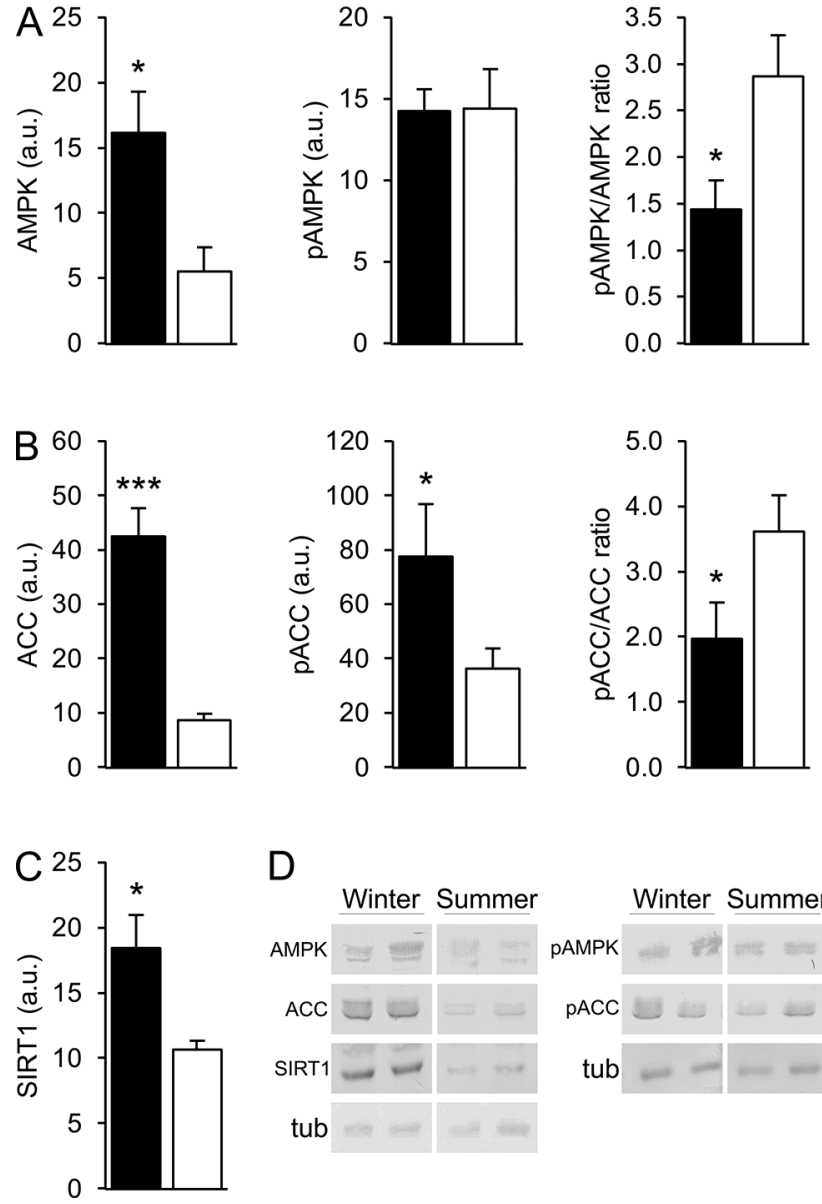

Figure 4. Expression of AMPK, ACC, and SIRT1 in the iBAT of winter- and summer-acclimatized Djungarian hamsters. Relative protein concentrations of total AMPK, phosphorylated AMPK (pAMPK), and pAMPK/AMPK ratio $(A)$; total ACC, phosphorylated ACC, and pACC/ACC ratio $(B)$; and total SIRT1 $(C)$. $D$, Representative Western blots for AMPK, pAMPK, ACC, pACC, SIRT1, and loading control $\alpha$ tubulin (tub). The results are expressed as mean arbitrary units (a.u.) + SEM of protein band intensities normalized to $\alpha$-tubulin. Asterisks indicate significant differences between the groups (two-tailed Student's $t$-test: one asterisk, $P<0.05$; three asterisks, $P<0.001) . N=16$ for the winter group (filled bars) and $N=8$ for the summer group (open bars). 


\section{$\underline{\mathrm{RF}}$ relative protein concentrations}
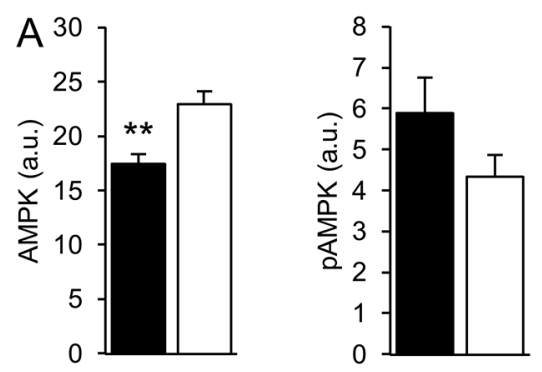

- Winter aSummer
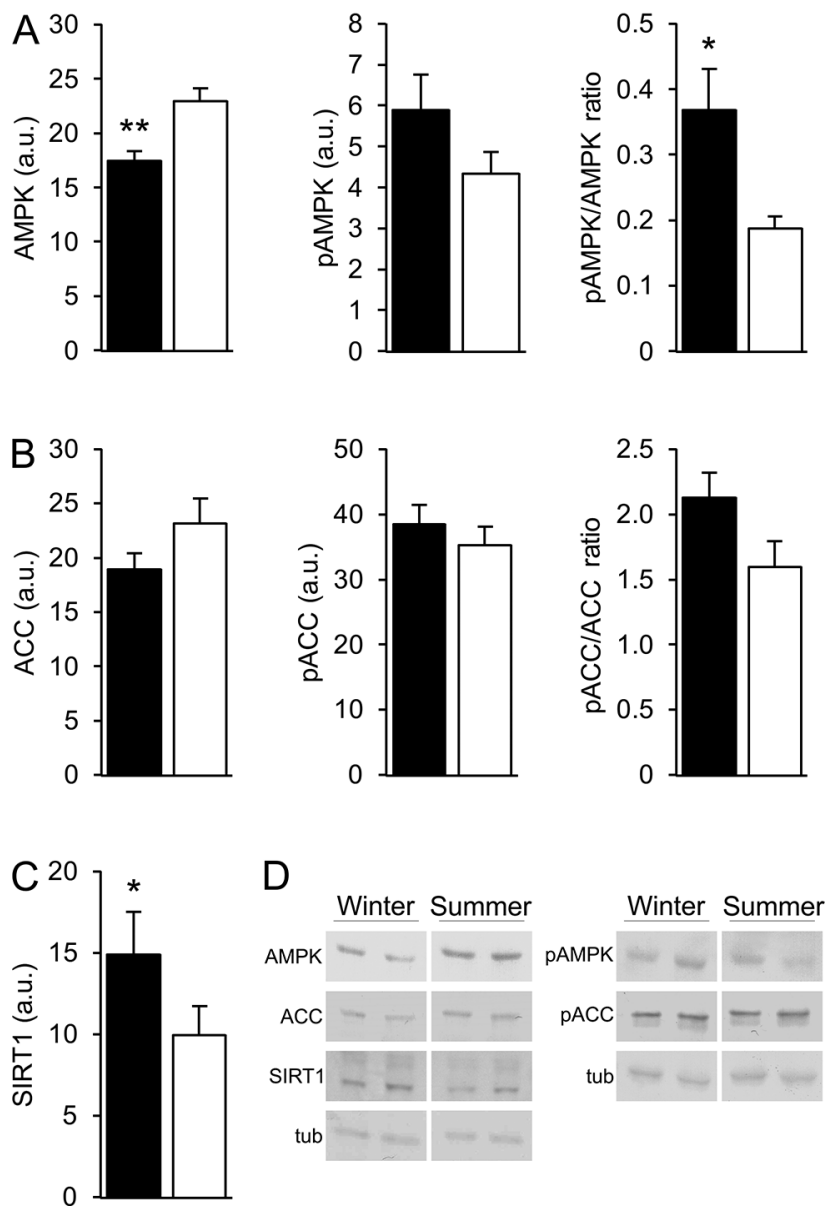

0

recycling associated with high-rate lipolysis (Gauthier et al. 2008). The more abundant amount of total AMPK and SIRT1 in sWAT and aWAT of the winter hamsters might contribute to efficient lipid mobilization and preservation of adipose tissue at the proper level prescribed by photoperiod. The low AMPK phosphorylation ratio observed in the winter group could indicate that AMPK function is downregulated during the euthermic phase, when hamsters forage and energy availability is not dependent on endogenous lipid reserves. Unfortunately, although all of our hamsters lost body mass during winter acclimatization, only a few animals entered daily torpor regularly. Hence, we were not able to measure protein levels from a sufficient amount of torpid animals to clarify whether the phosphorylation levels differ between the torpid and euthermic stages.

\section{HYPO relative protein concentrations}
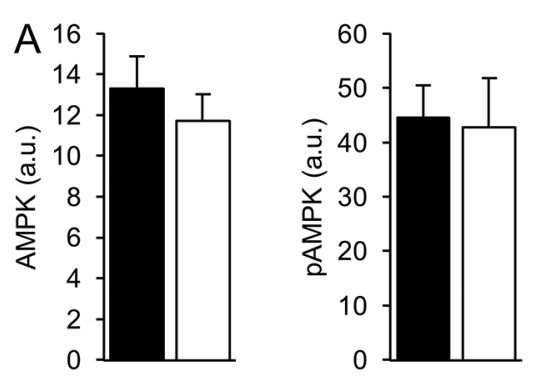

-Winter 口Summer
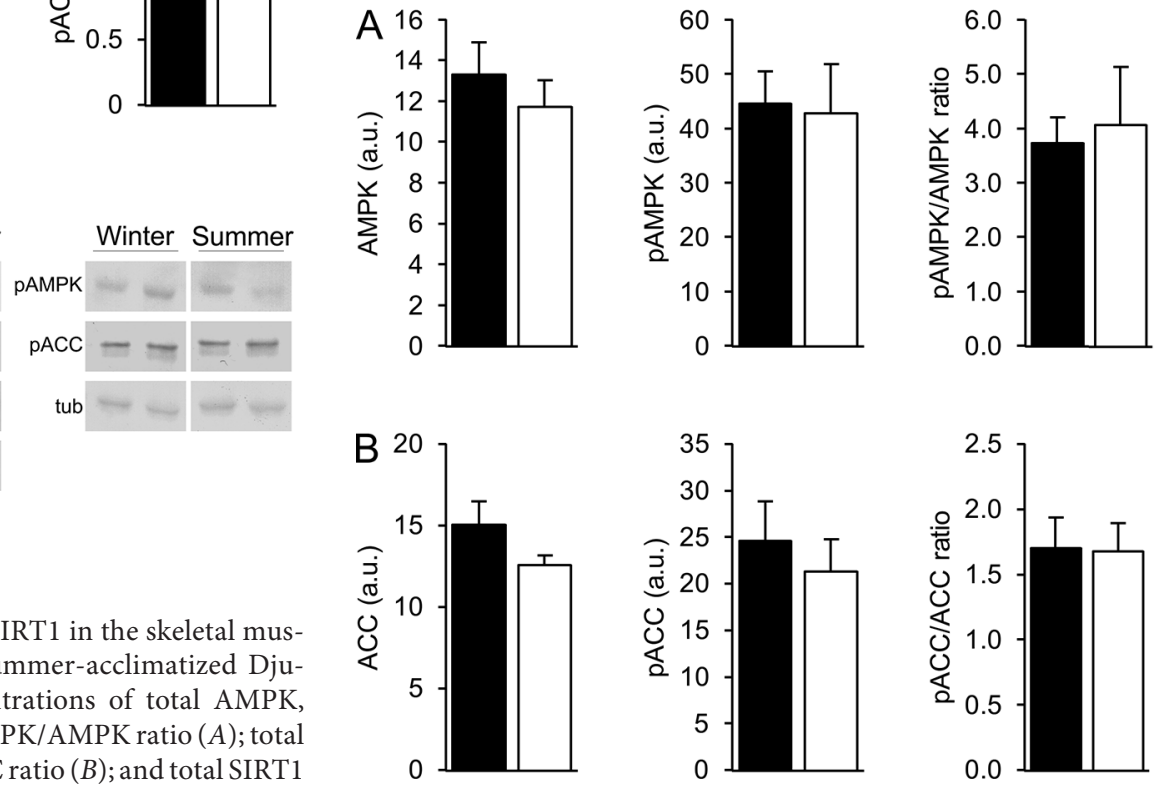

Figure 5. Expression of AMPK, ACC, and SIRT1 in the skeletal muscle (rectus femoris $[\mathrm{RF}]$ ) of winter- and summer-acclimatized Djungarian hamsters. Relative protein concentrations of total AMPK, phosphorylated AMPK (pAMPK), and pAMPK/AMPK ratio $(A)$; total ACC, phosphorylated ACC, and pACC/ACC ratio $(B)$; and total SIRT1 (C). D, Representative Western blots for AMPK, pAMPK, ACC, pACC, SIRT1, and loading control $\alpha$-tubulin (tub). The results are expressed as mean arbitrary units (a.u.) + SEM of protein band intensities normalized to $\alpha$-tubulin. Asterisks indicate significant differences between the groups (two-tailed Student's $t$-test: one asterisk, $P<0.05$; two asterisks, $P<0.01$ ). $N=16$ for the winter group (filled bars) and $N=8$ for the summer group (open bars).

SIRT1 are known to suppress adipocyte differentiation and lipogenesis in rats and mice (Sullivan et al. 1994; Habinowski and Witters 2001; Gaidhu et al. 2009). Furthermore, SIRT1 has been shown to increase lipolysis and fat mobilization in mouse WAT (Picard et al. 2004), whereas AMPK appears to have an antilipolytic effect that depends on the rate of lipolysis (Kim et al. 2016). AMPK is activated in adipocytes in response to increased lipolysis, for example, under fasting condition, and this activation subsequently inhibits the lipolytic pathway (Daval et al. 2005; Gauthier et al. 2008). The antilipolytic effect of AMPK in WAT has been proposed to function as a mechanism that limits energy depletion caused by excessive FA

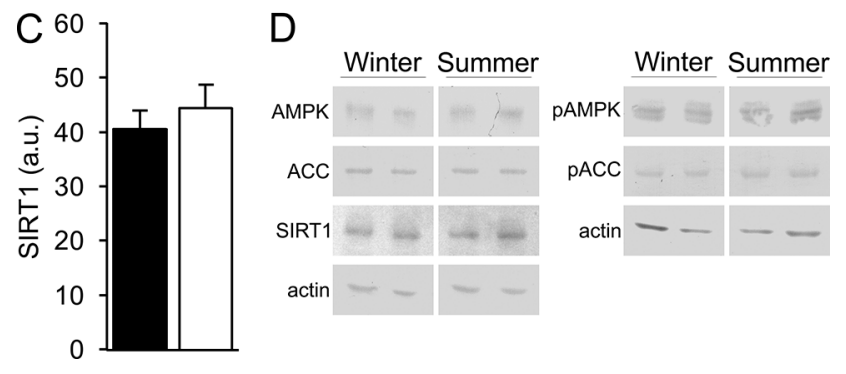

Figure 6. Expression of AMPK, ACC, and SIRT1 in the hypothalamus of winter- and summer-acclimatized Djungarian hamsters. Relative protein concentrations of total AMPK, phosphorylated AMPK (pAMPK), and $\mathrm{pAMPK} / \mathrm{AMPK}$ ratio $(A)$; total ACC, phosphorylated ACC, and pACC/ACC ratio $(B)$; and total SIRT1 $(C)$. D, Representative Western blots for AMPK, pAMPK, ACC, pACC, SIRT1, and loading control $\beta$ actin. The results are expressed as mean arbitrary units (a.u.) + SEM of protein band intensities normalized to $\beta$-actin. $N=16$ for the winter group (filled bars) and $N=8$ for the summer group (open bars). 
Interestingly, ACC expression was also higher in sWAT of the winter group, suggesting the potential for enhanced lipogenesis. However, a larger proportion of the total ACC was phosphorylated (inactive form) compared to that in the summer animals. ACC protein expression did not differ in aWAT between the groups, but the pACC level was more than twofold higher in the winter animals. Previous studies have shown that lipid mobilization is not evenly distributed in the short-dayexposed Djungarian hamsters. Large proportions of the lost fat mass are from the internally located fat pads, and the more external subcutaneous fat is utilized to a lesser extent (Bartness et al. 2002; Bowers et al. 2005). Presumably, sWAT has a more dynamic metabolic role as an insulating factor and lipid reserve during winter adaptation. Thus, the higher ACC protein level in sWAT of the winter hamsters could be relevant for the maintenance of the appropriate amount of subcutaneous lipid depots. In turn, aWAT is depleted to maintain the lean winter phenotype, and upregulation of the lipogenic pathway is not required. In addition, lower pACC levels in the summer animals indicate that WAT metabolism is directed toward lipogenesis as excepted since the Djungarian hamster increases body fat stores during summer (Heldmaier and Steinlechner 1981a; Klingenspor et al. 2000). The correlation between pAMPK and pACC was inconsistent in all tissues except aWAT, where a slight positive correlation was observed. Similar inconsistence has also been observed by previous studies in winter-acclimatized ground squirrels (Belke et al. 1998; Horman et al. 2005; Healy et al. 2011b; Lanaspa et al. 2015). It appears that ACC may be phosphorylated by AMPK-independent pathways during winter adaptation.

One distinct physiological response to short-day conditions in the Djungarian hamster is the increase in the thermogenic capacity of BAT (Heldmaier et al. 1981). It has to be noted that our experiment cannot distinguish between short photoperiod and cold acclimatization. Thus, the changes in protein expression levels are discussed as a result of winter adaptation in general. Chronic cold exposure has been shown to upregulate AMPK expression and activity in BAT of mice, suggesting that AMPK participates in long-term thermoregulation (Mulligan et al. 2007). In ground squirrels, expression of AMPK increases in BAT of hibernating animals compared to that in summertime controls (Horman et al. 2005). Our results show that also in the Djungarian hamster, AMPK expression is elevated in iBAT in response to winter acclimatization. High levels of ACC expression in the winter group (fivefold higher compared to the summer animals) indicate maintenance of efficient FA synthesis and renewal of the tissue in winter conditions. On the other hand, in summer, when the need for nonshivering thermogenesis is reduced, ACC expression and phosphorylation level are downregulated. Similar to sWAT and aWAT, SIRT1 expression was higher in iBAT of the winter hamsters. Studies in the Djungarian hamster have shown that short-day exposure increases PGC- $1 \alpha$ mRNA expression in BAT (Demas et al. 2002), and the number of mitochondria is increased 10-fold to enable the increase in nonshivering thermogenesis (Heldmaier et al. 1981). Both AMPK and SIRT1 positively regulate PGC1- $\alpha$, and increased expression of AMPK and SIRT1 in the winter- acclimatized hamsters might enhance the mitochondrial biogenesis and FA oxidation (Fulco and Sartorelli 2008; Ruderman et al. 2010). Overall, enhanced BAT activity is associated with increased expression and activity of AMPK and SIRT1 (Boutant et al. 2015; van Dam et al. 2015). Thus, higher expressions of AMPK, ACC, and SIRT1 are presumably associated with the increased thermogenic capacity of iBAT in response to winter acclimatization.

Winter acclimatization had less clear effects on skeletal muscle than on adipose tissues. In contrast to WAT and BBAT, AMPK expression declined in muscle of the winter group, and the pAMPK/AMPK ratio was higher compared to that in summer animals. Although not statistically significant, a similar pattern was observed in ACC expression and phosphorylation ( $P=0.07$ for $\mathrm{pACC} / \mathrm{ACC}$ ratio). However, similar to adipose tissues, SIRT1 expression was higher in muscle of winter hamsters. Both AMPK and SIRT1 increase FA oxidation and mitochondrial biogenesis in mouse and rat skeletal muscle (Zong et al. 2002; Lee et al. 2006; Gerhart-Hines et al. 2007). Higher AMPK phosphorylation ratio, along with higher SIRT1 expression, indicates elevated oxidative capacity in skeletal muscle of the winter-acclimatized hamsters, which could be beneficial during food shortages experienced in winter (e.g., during daily torpor), when muscles are forced to switch from carbohydrates to FA oxidation. Furthermore, transgenic mice overexpressing SIRT1 in skeletal muscles have reduced muscle mass and smaller muscle fiber (Chalkiadaki et al. 2014). Higher SIRT1 expression in muscle of the winter hamsters could contribute to the lower body mass.

Increased expression and activation of hypothalamic AMPK and SIRT1 increases food intake and body weight in mice (Minokoshi et al. 2004; Satoh et al. 2010). Reciprocally, inhibition of AMPK and SIRT1 has an opposite effect (Andersson et al. 2004; Çakir et al. 2009). Studies in marmots (Marmota flaviventris) and ground squirrels (Callospermophilus lateralis) suggest that suppression of hypothalamic AMPK activity is needed for the successful torpor and maintenance of hypophagia during hibernation season (Florant et al. 2010; Healy et al. 2011a). However, differing from deep hibernators, the Djungarian hamster does not become aphagic during winter. Instead, foraging and food intake are decreased to accommodate the lower body weight that remains in the limits of a certain set point determined by photoperiod (Heldmaier and Steinlechner 1981 $a$; Steinlechner et al. 1983). Furthermore, energetic balance is maintained despite the lower calorie intake (Scherbarth and Steinlechner 2010). Unchanged levels of hypothalamic AMPK, ACC, and SIRT1 indicate that the winter-acclimatized Djungarian hamsters were in an energetically balanced state. However, as stated previously, we did not compare the winter torpid and euthermic animals, and it is possible that hypothalamic AMPK, ACC, and SIRT1 expression and phosphorylation are altered between these stages.

To conclude, our results show that AMPK, ACC, and SIRT1 are associated with seasonal molecular adaptations in the energy metabolism of the Djungarian hamster. As hypothesized, expression levels differed between winter- and summer-acclimatized 
hamsters, observed distinctly as higher AMPK, ACC, and SIRT1 expression in WAT and iBAT of the winter group. This indicates that these proteins are involved in the adjustment of lipid reserves and increased thermogenic capacity of the winter-adapted Djungarian hamster. Although increased protein expression does not necessarily equal increased enzyme activity, higher AMPK and SIRT1 protein levels in different tissues of the Djungarian hamster may facilitate the metabolic adaptation for environmental and nutritional stresses the animals encounter in winter.

\section{Acknowledgments}

We thank Ms. Minna Orreveteläinen and Ms. Marja-Liisa MartimoHalmetoja for help with sampling and laboratory work. We also thank Mr. Jorma Toivonen for expert animal care and Mr. Matti Rauman for technical assistance. This study was financially supported by the Academy of Finland (grant 134048 to S.S.) and by the Emil Aaltonen Foundation (personal working grant to S.M.E.K).

\section{Literature Cited}

Andersson U., K. Filipsson, C.R. Abbott, A. Woods, K. Smith, S.R. Bloom, D. Carling and C.J. Small. 2004. AMP-activated protein kinase plays a role in the control of food intake. J Biol Chem 279:12005-12008.

Bartness T.J., G.E. Demas, and C. Kay Song. 2002. Seasonal changes in adiposity: the roles of the photoperiod, melatonin and other hormones, and sympathetic nervous system. Exp Biol Med 227:363-376.

Belke D.D., L.C.H. Wang, and G.D. Lopaschuk. 1998. AcetylCoA carboxylase control of fatty acid oxidation in hearts from hibernating Richardson's ground squirrels. Biochim Biophys Acta 1391:25-36.

Boutant M., M. Joffraud, S.S. Kulkarni, E. García-Casarrubios, P.M. García-Roves, J. Ratajczak, P.J. Fernández-Marcos, A.M. Valverde, M. Serrano, and C. Cantó. 2015. SIRT1 enhances glucose tolerance by potentiating brown adipose tissue function. Mol Metab 4:118-131.

Bowers R.R., T.W. Gettys, V. Prpic, R.B.S. Harris, and T.J. Bartness. 2005. Short photoperiod exposure increases adipocyte sensitivity to noradrenergic stimulation in Siberian hamsters. Am J Physiol 288:R1354-R1360.

Çakir I., M. Perello, O. Lansari, N.J. Messier, C.A. Vaslet, and E.A. Nillni. 2009. Hypothalamic SIRT1 regulates food intake in a rodent model system. PLoS ONE 4:e8322.

Chalkiadaki A., M. Igarashi, A.S. Nasamu, J. Knezevic, and L. Guarente. 2014. Muscle-specific SIRT1 gain-of-function increases slow-twitch fibers and ameliorates pathophysiology in a mouse model of Duchenne muscular dystrophy. PLoS Genet 10:e1004490.

Daval M., F. Diot-Dupuy, R. Bazin, I. Hainault, B. Viollet, S. Vaulont, E. Hajduch, P. Ferré, and F. Foufelle. 2005. Anti- lipolytic action of AMP-activated protein kinase in rodent adipocytes. J Biol Chem 280:25250-25257.

Demas G.E. and T.J. Bartness. 2001. Direct innervation of white fat and adrenal medullary catecholamines mediate photoperiodic changes in body fat. Am J Physiol 281:R1499-R1505.

Demas G.E., R.R. Bowers, T.J. Bartness, and T.W. Gettys. 2002. Photoperiodic regulation of gene expression in brown and white adipose tissue of Siberian hamsters (Phodopus sungorus). Am J Physiol 282:R114-R121.

Florant G.L., A.M. Fenn, J.E. Healy, G.K. Wilkerson, and R.J. Handa. 2010. To eat or not to eat: the effect of AICAR on food intake regulation in yellow-bellied marmots (Marmota flaviventris). J Exp Biol 213:2031-2037.

Fulco M. and V. Sartorelli. 2008. Comparing and contrasting the roles of AMPK and SIRT1 in metabolic tissues. Cell Cycle 7:3669-3679.

Gaidhu M.P., S. Fediuc, N.M. Anthony, M. So, M. Mirpourian, R.L.S. Perry, and R.B. Ceddia. 2009. Prolonged AICARinduced AMP-kinase activation promotes energy dissipation in white adipocytes: novel mechanisms integrating HSL and ATGL. J Lipid Res 50:704-715.

Gauthier M.-S., H. Miyoshi, S.C. Souza, J.M. Cacicedo, A.K. Saha, A.S. Greenberg, and N.B. Ruderman. 2008. AMPactivated protein kinase is activated as a consequence of lipolysis in the adipocyte: potential mechanism and physiological relevance. J Biol Chem 283:16514-16524.

Gerhart-Hines Z., J.T. Rodgers, O. Bare, C. Lerin, S.-H. Kim, R. Mostoslavsky, F.W. Alt, Z. Wu, and P. Puigserver. 2007. Metabolic control of muscle mitochondrial function and fatty acid oxidation through SIRT1/PGC-1a. EMBO J 26: 1913-1923.

Habinowski S.A. and L.A. Witters. 2001. The effects of AICAR on adipocyte differentiation of 3T3-L1 cells. Biochem Biophys Res Commun 286:852-856.

Haigis M.C. and D.A. Sinclair. 2010. Mammalian sirtuins: biological insights and disease relevance. Annu Rev Pathol Mech Dis 5:253-295.

Hardie D.G., F.A. Ross, and S.A. Hawley. 2012. AMPK: a nutrient and energy sensor that maintains energy homeostasis. Nat Rev Mol Cell Biol 13:251-262.

Healy J.E., J.L. Bateman, C.E. Ostrom, and G.L. Florant. $2011 a$. Peripheral ghrelin stimulates feeding behavior and positive energy balance in a sciurid hibernator. Horm Behav 59:512519.

Healy J.E., C.N. Gearhart, J.L. Bateman, R.J. Handa, and G.L. Florant. 2011b. AMPK and ACC change with fasting and physiological condition in euthermic and hibernating goldenmantled ground squirrels (Callospermophilus lateralis). Comp Biochem Physiol A 159:322-331.

Heldmaier G. and S. Steinlechner. 1981a. Seasonal control of energy requirements for thermoregulation in the Djungarian hamster (Phodopus sungorus), living in natural photoperiod. J Comp Physiol B 142:429-437.

- 1981b. Seasonal pattern and energetics of short daily torpor in the Djungarian hamster, Phodopus sungorus. Oecologia 48:265-270. 
Heldmaier G., S. Steinlechner, J. Rafael, and P. Vsiansky. 1981. Photoperiodic control and effects of melatonin on nonshivering thermogenesis and brown adipose tissue. Science 212:917-919.

Horman S., N. Hussain, S.M. Dilworth, K.B. Storey, and M.H. Rider. 2005. Evaluation of the role of AMP-activated protein kinase and its downstream targets in mammalian hibernation. Comp Biochem Physiol B 142:374-382.

Kahn B.B., T. Alquier, D. Carling, and D.G. Hardie. 2005. AMPactivated protein kinase: ancient energy gauge provides clues to modern understanding of metabolism. Cell Metab 1:15-25.

Kim S.-J., T. Tang, M. Abbott, J.A. Viscarra, Y. Wang, and H.S. Sula. 2016. AMPK phosphorylates desnutrin/ATGL and hormone-sensitive lipase to regulate lipolysis and fatty acid oxidation within adipose tissue. Mol Cell Biol 36:1961-1976.

Kinnunen S., S. Mänttäri, K.-H. Herzig, P. Nieminen, A.-M. Mustonen, and S. Saarela. 2016. Effects of wintertime fasting and seasonal adaptation on AMPK and ACC in hypothalamus, adipose tissue and liver of the raccoon $\operatorname{dog}$ (Nyctereutes procyonoides). Comp Biochem Physiol A 192:44-51.

Klingenspor M., H. Niggemann, and G. Heldmaier. 2000. Modulation of leptin sensitivity by short photoperiod acclimation in the Djungarian hamster, Phodopus sungorus. J Comp Physiol B 170:37-43.

Knopper L.D. and P. Boily. 2000. The energy budget of captive Siberian hamsters, Phodopus sungorus, exposed to photoperiod changes: mass loss is caused by a voluntary decrease in food intake. Physiol Biochem Zool 73:517-522.

Lanaspa M.A., L.E. Epperson, N. Li, C. Cicerchi, G.E. Garcia, C.A. Roncal-Jimenez, J. Trostel, et al.2015. Opposing activity changes in AMP deaminase and AMP-activated protein kinase in the hibernating ground squirrel. PLoS ONE 10:e0123509.

Lee W.J., M. Kim, H.S. Park, H.S. Kim, M.J. Jeon, K.S. Oh, E.H. Koh, et al. 2006. AMPK activation increases fatty acid oxidation in skeletal muscle by activating PPAR $\alpha$ and PGC1. Biochem Biophys Res Commun 340:291-295.

Minokoshi Y., T. Alquier, H. Furukawa, Y.-B. Kim, A. Lee, B. Xue, J. Mu, et al. 2004. AMP-kinase regulates food intake by responding to hormonal and nutrient signals in the hypothalamus. Nature 428:569-574.

Mulligan J.D., A.A. Gonzalez, A.M. Stewart, H.V. Carey, and K.W. Saupe. 2007. Upregulation of AMPK during cold exposure occurs via distinct mechanisms in brown and white adipose tissue of the mouse. J Physiol 580:677-684.

Picard F., M. Kurtev, N. Chung, A. Topark-Ngarm, T. Senawong, R.M. De Oliveira, M. Leid, M.W. McBurney, and L. Guarente.
2004. SIRT1 promotes fat mobilization in white adipocytes by repressing PPAR- $\gamma$. Nature 429:771-776.

Rodgers J.T., C. Lerin, W. Haas, S.P. Gygi, B.M. Spiegelman, and P. Puigserver. 2005. Nutrient control of glucose homeostasis through a complex of PGC-1a and SIRT1. Nature 434:113-118.

Rouble A.N. and K.B. Storey. 2015. Characterization of the SIRT family of NAD+-dependent protein deacetylases in the context of a mammalian model of hibernation, the thirteen-lined ground squirrel. Cryobiology 71:334-343.

Ruderman N.B., X.J. Xu, L. Nelson, J.M. Cacicedo, A.K. Saha, F. Lan, and Y. Ido. 2010. AMPK and SIRT1: a long-standing partnership? Am J Physiol 298:E751-E760.

Satoh A., C.S. Brace, G. Ben-Josef, T. West, D.F. Wozniak, D.M. Holtzman, E.D. Herzog, and S.-I. Imai. 2010. SIRT1 promotes the central adaptive response to diet restriction through activation of the dorsomedial and lateral nuclei of the hypothalamus. J Neurosci 30:10220-10232.

Scherbarth F. and S. Steinlechner. 2010. Endocrine mechanisms of seasonal adaptation in small mammals: from early results to present understanding. J Comp Physiol B 180:935952.

Steinlechner S., G. Heldmaier, and H. Becker. 1983. The seasonal cycle of body weight in the Djungarian hamster: photoperiodic control and the influence of starvation and melatonin. Oecologia 60:401-405.

Sullivan J.E., K.J. Brocklehurst, A.E. Marley, F. Carey, D. Carling, and R.K. Beri. 1994. Inhibition of lipolysis and lipogenesis in isolated rat adipocytes with AICAR, a cellpermeable activator of AMP-activated protein kinase. FEBS Lett 353:33-36.

Suter M., U. Riek, R. Tuerk, U. Schlattner, T. Wallimann, and T. Neumann. 2006. Dissecting the role of 5'-AMP for allosteric stimulation, activation, and deactivation of AMPactivated protein kinase. J Biol Chem 281:32207-32216.

van Dam A.D., S. Kooijman, M. Schilperoort, P.C.N. Rensen, and M.R. Boon. 2015. Regulation of brown fat by AMPactivated protein kinase. Trends Mol Med 21:571-579.

Wade G.N. and T.J. Bartness. 1984. Effects of photoperiod and gonadectomy on food intake, body weight, and body composition in Siberian hamsters. Am J Physiol 246:R26-R30.

Zong H., J.M. Ren, L.H. Young, M. Pypaert, J. Mu, M.J. Birnbaum, and G.I. Shulman. 2002. AMP kinase is required for mitochondrial biogenesis in skeletal muscle in response to chronic energy deprivation. Proc Natl Acad Sci USA 99: 15983-15987. 